\title{
Correspondence
}

Editor: Greg Wilkinson

\section{A Case of Resistant Schizophrenia}

SIR: It is gratifying that our colleagues at the Joint Hospital (Roberts et al, Journal, December 1986, 149, 789-793) have discovered neuroleptic-resistant schizophrenia. However, had they visited any of the large psychiatric hospitals, which they seem to prefer to call asylums, they would be likely to find a number of patients with this disorder.

Notwithstanding the failure of electroconvulsive therapy in this patient, I would suggest that lithium should be tried. Sometimes "continuously floridly psychotic behaviour' has a masked affective component, as Crammer suggests in the paper.

We have reported the case of a 27 -year-old married lady who required high doses of depot neuroleptics to control episodes of schizophrenia (as diagnosed at that time) associated with very disturbed behaviour and to maintain prophylaxis (Barnes \& Bridges, 1980). Her severe symptoms were improved, but certainly not controlled, by the use of ECT. However, there was a good response to lithium, although, ultimately, she was found to be most settled on haloperidol $(20 \mathrm{mg}$ t.d.s.) and carbamazepine $(400 \mathrm{mg}$ t.d.s.) with procyclidine. Her compliance on this medication is good, and it has kept her out of hospital so far for the past four years. I should add that she was transferred originally to my care at Bexley Hospital from the Bethlem Royal Hospital, where it was considered that she was doing too much damage to both the staff and the fabric and hence "asylum" was needed.

Division of Psychiatry
Guy's Hospital

P. K. BRIDGES

London SE1 9RT

\section{Reference}

BARNES, T. R. E. \& Bridges, P. K. (1980) British Medical Journal, ii, 274-275.

\section{Anorexia Nervosa and Dysmorphophobia}

SIR: I should like to comment upon Sturmey \& Slade's paper (Journal, December 1986, 149, 780782). Anorexia nervosa and dysmorphophobia are functional disorders which usually occur in young individuals and are characterised by disturbances of the body image. In the former condition subjects characteristically perceive themselves as fatter than they are, as with the reported patient who "considered herself to be generally too 'broad"', whereas in dysmorphophobia, subjects typically complain about one particular aspect of their appearance, such as the nose, chin, mouth, breasts, or penis (Morselli, 1886; Hay, 1970; Thomas, 1984).

In anorexia nervosa and in the reported patient weight loss occurs, and consequently observed appearance cannot be considered normal. In dysmorphophobia there is a subjective feeling of ugliness or physical defect which the patient feels is noticeable to others, although appearance is within normal limits (Morselli, 1886).

I think that it is incorrect to consider that their patient exhibits dysmorphophobia, as the disturbance in body image was not localised, abnormality of body size was present, and there was a primary diagnosis of anorexia nervosa from which the dissatisfaction with appearance developed.

Making these distinctions is important if one wishes to identify and study a homogenous group of dysmorphophobies where an over-valued idea concerning appearance, in the absence of underlying classifiable psychiatric disease, is the principal abnormality.

\section{University Hospital of South Manchester}

Christopher S. ThOMAS Manchester M20 8LR

\section{References \\ HAY, G. G. (1970) Dysmorphophobia. British Journal of Psychiatry. $116,399-406$. \\ Morsell, E. (1886) Sulla dismorfofobia e sulla tafefobia. Bolletino Accademia delle Scienze Mediche di Genova, 6, 100-119. \\ Tномия, C. S. (1984) Dysmorphophobia: a question of definition. British Journal of Psychiatry, 144, 513-516.}

SIR: The report on anorexia nervosa and dysmorphophobia (Sturmey \& Slade, Journal, December $1986,149,780-782$ ) demonstrates how easy it is to further complicate psychiatric nosology.

Certainly the history suggests that the patient warrants a diagnosis of anorexia nervosa; many of the classical features are present, including a disturbed body image. Dysmorphophobia, however, is confused yet again by an initial emphasis on physical 